\title{
Avaliação do temperamento afetivo e emocional em estudantes universitários da
}

\section{metodologia ativa de ensino}

\author{
Evaluation of affective and emotional temperament in university students of the active teaching \\ methodology
}

Evaluación del temperamento afectivo y emocional en estudiantes universitarios de la metodología de enseñanza activa

Isadora Archanjo Ferreira Braga ORCID: https://orcid.org/0000-0002-7080-1693 Universidade Federal de Sergipe, Brasil E-mail: isadoraarchanjo@hotmail.com Lucas Ribeiro Maroneze ORCID: https://orcid.org/0000-0002-5689-3245 Universidade Federal de Sergipe, Brasil E-mail: lucasmaroneze@gmail.com

Kelly Virgínia Gabriel de Carvalho ORCID: https://orcid.org/0000-0003-2326-6277 Universidade Federal de Sergipe, Brasil

E-mail: kelly..carvalho@academico.ufs.br

Fábio Rodrigues de Moura

ORCID: https://orcid.org/0000-0002-6532-110X Universidade Federal de Sergipe, Brasil

E-mail: fabiromoura@gmail.com

Priscila Lima dos Santos

ORCID: https://orcid.org/0000-0002-8863-5718 Universidade Federal de Sergipe, Brasil E-mail: plimabio@gmail.com

Debora Dos Santos Tavares

ORCID: https://orcid.org/0000-0001-5452-0771 Universidade Federal de Sergipe, Brasil

E-mail: tavaresdebora@gmail.com

\begin{abstract}
Resumo
A metodologia ativa é uma construção pedagógica que utiliza fatos reais ou fictícios como estratégia de ensinoaprendizagem, com o objetivo de refletir sobre uma situação, com diferentes possibilidades de análise, e então solucionar impasses. Entendendo que essas experiências fazem parte da construção da personalidade do sujeito, surge a ideia de que os desafios desse método de ensino poderiam interferir nas dimensões temperamentais afetivas e emocionais dos estudantes. Assim, o presente estudo avaliou o temperamento afetivo e emocional dos estudantes matriculados do primeiro ano da Universidade Federal de Sergipe (Campus Universitário Professor Antônio Garcia Filho), a qual utiliza diferentes metodologias ativas, especialmente a Aprendizagem Baseada em Problemas (ABP). Observou-se impacto negativo da ABP no temperamento emocional dos estudantes (vontade, controle, coping, sensibilidade e o indicador Funcionamento Emocional Global). Entretanto, verificou-se aumento nas dimensões desinibição e euforia após envolvimento com a metodologia ativa aplicada. Portanto, considera-se que os aspectos da metodologia ativa podem contribuir positiva ou negativamente em determinados aspectos do temperamento afetivo e emocional, sendo importante outros estudos em diferentes academias, bem como a comparação com outras metodologias de ensino vigentes, para atestar os efeitos da metodologia ativa no desenvolvimento temperamental.
\end{abstract}

Palavras-chave: Temperamento afetivo; Metodologia ativa; Temperamento emocional; Ensino.

\begin{abstract}
Active methodology is a pedagogical method that uses real or fictitious facts as a teaching-learning strategy, aiming to reflect on a situation that has different possibilities for analysis to solvethe focused issue. Considering that these experiences are part of the construction of the subject's personality, the challenges faced by this teaching method could interfere in the students' affective and emotional temperamental dimensions. Thus, the present study evaluated the affective and emotional temperament of students enrolled in the first year of the Federal University of Sergipe (Campus Universitário Professor Antônio Garcia Filho), which uses different active methodologies, especially
\end{abstract}


Problem Based Learning (PBL). There was a negative impact of PBL on students' emotional temperament (willingness, control, coping, sensitivity and Global Emotional Functioning indicator). However, there was an increase/favoring of disinhibition and euphoria after the active methodology experience by the students. Hence, theactive methodology inherents aspects can contribute positively or negatively in certain dimensions of the affective and emotional temperament. Nonetheless, other studies in different academies, as well as the comparison with other current teaching methodologies are important to attest the effect of active methodology on temperament.

Keywords: Affective temperament; Active methodology; Emotional temperament; Teaching.

\section{Resumen}

La metodología activa es una construcción pedagógica que utiliza hechos reales o ficticios como estrategia de enseñanza-aprendizaje, con el objetivo de reflexionar sobre una situación, con diferentes posibilidades de análisis, para luego resolver puntos muertos. Entendiendo que estas experiencias son parte de la construcción de la personalidad del sujeto, surge la idea de que los desafíos de este método de enseñanza podrían interferir en las dimensiones temperamental afectiva y emocional de los estudiantes. Así, el presente estudio evaluó el temperamento afectivo y emocional de los estudiantes matriculados en el primer año de la Universidad Federal de Sergipe (Campus Universitário Professor Antônio Garcia Filho), que utiliza diferentes metodologías activas, especialmente el Aprendizaje Basado en Problemas (ABP). Hubo un impacto negativo del ABP en el temperamento emocional de los estudiantes (voluntad, control, coping, sensibilidad y el indicador de Funcionamiento Emocional Global). Sin embargo, hubo un aumento en las dimensiones de desinhibición y euforia luego de involucrarse con la metodología activa aplicada. Por tanto, se considera que los aspectos de la metodología activa pueden contribuir positiva o negativamente en determinados aspectos del temperamento afectivo y emocional, siendo importantes otros estudios en diferentes academias, así como la comparación con otras metodologías docentes actuales, para atestiguar los efectos de la metodología activa en el desarrollo temperamental.

Palabras clave: Temperamento afectivo; Metodología activa; Temperamento emocional; Ensenãnza.

\section{Introdução}

O sistema de educação no ensino superior utiliza a metodologia conservadora na maioria das universidades brasileiras, a qual apresenta muitas vezes uma insuficiente articulação educacional para enfrentar as conjunturas encontradas na prática, o que demonstra a necessidade de estabelecer novas estratégias pedagógicas de ensino superior na área da saúde que formem profissionais com domínio técnico-científico e com habilidades necessárias no campo de trabalho (Fernandes et al., 2003). Nesta perspectiva, a metodologia ativa, por meio da Metodologia da Problematização, Aprendizagem Baseada em Problemas (ABP) e Aprendizagem Baseada em Equipes (ABE), apresenta-se como uma estratégia promissora para estabelecer a articulação entre a universidade, serviço e comunidade (Mitre et al., 2008).

Nessas propostas, o docente exerce um papel de coadjuvante, ao organizar e facilitar a construção do conhecimento técnico-científico, e o discente ocupa o papel de autor principal no desenvolvimento do seu aprendizado, dispondo de ferramentas capazes de estimular o planejamento, produção, organização, proposição e análise de ideias para fomentar ações socialmente relevantes, a partir de uma situação problema (Ventura et al, 2020, Saupe et al., 2011, Berbel, 1995). De acordo com Neufeld et al (2020), a ABP estimula a agilidade e sensatez na tomada de decisão em ambiente acadêmico, direcionando os discentes a uma melhor atuação no espaço profissional, por experimentar situações próximas à realidade.

Entretanto, para que o método seja devidamente proveitoso, os acadêmicos envolvidos precisam desenvolver habilidades de comunicação e discussão, bem como utilizar-se de atitudes cooperativas e colaborativas para manter a boa convivência entre os componentes do grupo, sendo competências intimamente atreladas à personalidade do indivíduo (Jang; Park, 2016).

Partindo deste princípio e tendo em vista o papel significante do temperamento na formação da personalidade (Akiskal, 1998; Cloninger; Svrakic; Przybeck, 1993) e a suscetibilidade da mente de um indivíduo sofrer mudanças à medida que vivencia experiências diversas (Lara et al., 2012), o convívio rotineiro com os desafios impostos pela metodologia ativa de ensino-aprendizagem poderia, ao longo de um ano, influenciar no temperamento emocional e afetivo do estudante.

Os aspectos temperamentais possuem uma base biológica marcante, mas sofrem influências do meio e englobam aspectos cognitivos, comportamentais e emocionais, atrelados à formação da personalidade. (LARA, 2011, Cloninger, Svrakic, 
Przybeck, 1993). Beck (2013), ao pontuar sobre a Teoria Cognitiva, expõe sobre a influência das emoções na construção dos pensamentos e na formação dos comportamentos perante as situações do dia a dia, sejam elas estressoras ou não, a depender de como a pessoa as interpreta, fortemente estimulada pela personalidade construída desde a infância, com crenças aprofundadas sobre si mesma. A rotina acadêmica, com as exigências que a metodologia ativa estabelece, pode afetar as emoções, e, consequentemente, o modo de pensar e de agir, atuando na melhoria ou piora da qualidade de vida dos estudantes (Torres; Sampaio; Caldeira, 2019).

Segundo Lara (2011), o temperamento obedece a duas principais dimensões: emocional e afetiva. A primeira se refere aos aspectos mais relacionais, correspondendo à maneira intrínseca que cada pessoa interage com as experiências exteriores, de maneira apática, evitativa, disfórica, obsessiva, volátil, eutímica, hipertímica, eufórica, desinibida, irritável, depressiva ou ciclotímica (Lara et al., 2012, Lara, 2011). A segunda se refere aos sentimentos de desejo, raiva, inibição, sensibilidade, coping, controle e vontade, podendo ser correlacionados algumas doenças psiquiátricas, especificamente aos transtornos de humor, quando percebido alteração em uma ou mais esferas (Lara et al., 2012; Lara, 2011).

Diante do exposto, o presente estudo se propôs a avaliar o temperamento afetivo e emocional de estudantes ingressos na Universidade Federal de Sergipe que emprega, na sua grade curricular, a metodologia ativa de ensino. Foi possível observar a evolução do temperamento dos estudantes, na expectativa de promover discussões e melhorar a relação estudante (seu temperamento e afetividade) - universidade (seus métodos de ensino-aprendizagem).

\section{Metodologia}

\subsection{Local e tipo de pesquisa}

Trata-se de um estudo descritivo prospectivo realizado na Universidade Federal de Sergipe (UFS), Campus Universitário Professor Antônio Garcia Filho, em Sergipe (Brasil). A pesquisa foi empregada em dois momentos: no início (primeira fase) e no final do primeiro ano (segunda fase), a fim de avaliar o temperamento afetivo e emocional dos discentes no primeiro ano do ensino superior em contato com a metodologia ativa de ensino-aprendizagem. Foi utilizado o método quantitativo, que permite a análise matemática de conjuntos de dados através de porcentagem e técnicas estatísticas, possibilitando uma visão mais acurada dos resultados do estudo (Pereira et al., 2018).

\subsection{População do estudo}

A amostra foi constituída por acadêmicos do primeiro ano do campus da UFS, sem contato com alguma metodologia ativa e que residem no estado de Sergipe. Não foi realizada análise por curso, visto que a instituição emprega a prática pedagógica citada em todas as carreiras e no primeiro ano, todos os estudantes vivenciam apenas disciplinas comuns aos profissionais de saúde, ofertadas pelo Departamento de Educação em Saúde (DESL) da referida universidade.

Entretanto, por considerar que alguns fatores podem interferir no quadro temperamental do indivíduo (LARA, 2011), excluiu-se os usuários de medicamentos ou outros tipos de substâncias que interferem no comportamento neuropsíquico ou na capacidade de cognição; os estudantes que já tiveram contato anteriormente - tanto como docente quanto discente - com a metodologia ativa, inclusive os que estão cursando novamente o primeiro ano; os indivíduos sensibilizados com problemas familiares ou com entes queridos; os acadêmicos com o diagnóstico de doenças psiquiátricas; e os alunos que não participaram de uma das etapas da pesquisa.

\subsection{Coleta de dados}

A presente pesquisa segue a Resolução 510/16 do Conselho Nacional de Saúde (2016) e foi aprovada pelo Comitê de Ética em Pesquisa em Seres Humanos da UFS sob o número 2.537.985. Foram coletados dados sociodemográficos, bem como 
estado de residência antes do início da graduação.

O questionário AFECTS foi utilizado para avaliar a evolução temperamental (LARA et al., 2012). O instrumento possui mais de 60 itens que permitem obter informações individuais sobre os temperamentos emocional e afetivo. A seção emocional possui sete dimensões (desejo, raiva, inibição, sensibilidade, coping/resiliência, controle e vontade), sendo que seis apresentam oito perguntas, excluindo-se a dimensão do desejo, a qual apresenta quatro perguntas. Cada dimensão contém perguntas com uma pontuação de 1 a 7, gerando um valor total que varia de 8 a 56 . A exceção é atribuída ao temperamento desejo, em que a soma final está incluída no intervalo de 4 a 28, visto que possui somente quatro perguntas.

Além disso, o indicador Funcionamento Emocional Global (GEF) foi utilizado para estabelecer uma visão mais sintética do temperamento emocional nas duas fases, a partir das correlações positivas e negativas das dimensões. Foi calculado pela seguinte fórmula: GEF $=$ Vontade + Controle + Coping + Cautela (faceta da inibição presente nas 4 perguntas finais) - Raiva - Sensibilidade - Medo (faceta da inibição presente nas 4 perguntas iniciais) + 60 (intervalo de pontuação de -52 a 236$)$.

A afetiva emprega a escala de Likert, na qual se atribui uma escala intervalar de um a cinco para cada pergunta dos doze temperamentos afetivos, organizados na seguinte ordem: depressivo (ou melancólico), ansioso (ou evitativo), apático, cíclotímico, disfórico, volátil, obsessivo, eutímico, hipertímico, irritável, desinibido e eufórico. Tais dimensões foram agrupadas em quatro padrões, a saber, o internalizante, externalizante, instável e estável, sendo que cada um representa a junção de três variáveis. Assim, o grupo internalizante engloba os temperamentos melancólico, evitativo e apático, enquanto o externalizante inclui eufórico, desinibido e irritável. Os temperamentos ciclotímico, disfórico e volátil formam o grupo instável e o estável, sendo composto pelos estados eutímico, hipertímico e obsessivo.

Em relação à afetiva, a seção possui doze perguntas, cada uma relacionada a um temperamento. Há cinco itens possíveis para resposta, que partem de 1 a 5 pontos, correspondendo de "nada como eu" até "exatamente como eu", conforme o grau de identificação com a dimensão. As médias finais para cada uma das doze variáveis foram calculadas, no qual representam um comparativo entre o início e final do ano.

Além disso, foram calculadas as médias da somatória final para cada um dos quatros sentimentos agrupados (internalizante, externalizante, instável e estável) nos dois momentos (fase 1 e fase 2). Para tal, obteve-se o valor da soma das perguntas das três variáveis que formam cada padrão, com a pontuação final variando de 3 a 15. Ressalta-se que quanto mais próximo dos 3 pontos, maior é a não identificação com o padrão ("nada como eu”), enquanto a proximidade aos 15 pontos relaciona-se a maior reconhecimento ("exatamente como eu") (LARA et al., 2012).

\subsection{Análise Estatística}

As informações obtidas foram tabuladas no software EXCEL Microsoft Excel (2016) e analisadas no GraphPadPrism, versão 7.0 (2005). Na análise descritiva foram utilizadas as frequências absolutas e relativas. O somatório final das dimensões dos temperamentos emocionais e afetivos são as variáveis dependentes, exibidos na forma de gráficos, com o intuito de verificar as modificações temperamentais dos estudantes após o contato com a metodologia ativa. Por se tratar de dados não paramétricos, para a análise inferencial, seguiu-se com a comparação entre as médias dos grupos utilizando o teste de MannWhitney, com intervalo de confiança de $95 \%$. Avaliou-se a relação de cada dimensão do temperamento emocional e afetivo, bem como do GEF com as variáveis sexo e mudança de estado, em relação ao início e fim do primeiro ano letivo. 


\section{Resultados}

\subsection{Caracterização da amostra}

A amostra foi composta por 101 acadêmicos matriculados no primeiro ano da instituição de ensino e participantes das duas fases da pesquisa. Observou-se que 18,81\% $(n=19)$ dos discentes são originados de outros estados e que $64,4 \%$ (n=65) são do sexo feminino.

\subsection{Temperamento Emocional}

Em uma avaliação global, é perceptível o aumento da média na dimensão sensibilidade $(34,7$ para 36,$64 ; \mathrm{p}=0,008)$ (Gráfico 1) ao longo do primeiro ano. A dimensão controle exibiu uma redução da média entre as duas etapas (44,72 para 41,49; $\mathrm{p}=<0,0001$ ) (Gráfico 1). De forma semelhante, verifica-se uma redução da média da vontade (43,67 para 40,52; p= $<0,0001$ ) (Gráfico 1). Concomitante a isso, foi averiguado uma redução da média do coping $(43,4$ para 41,95; p=0,0465) (Gráfico 1). No entanto, na segunda fase, as quatro dimensões evidenciaram médias consideradas altas. Não houve diferença quanto às variáveis desejo, raiva e inibição. Um outro indicador empregado baseou-se nas correlações positivas e negativas dos temperamentos: o GEF, que apresentou redução da sua média (130,7 para 120,4; p=0,0001) (Gráfico 2).

Gráfico 1: Pontuação média de cada dimensão do temperamento emocional.

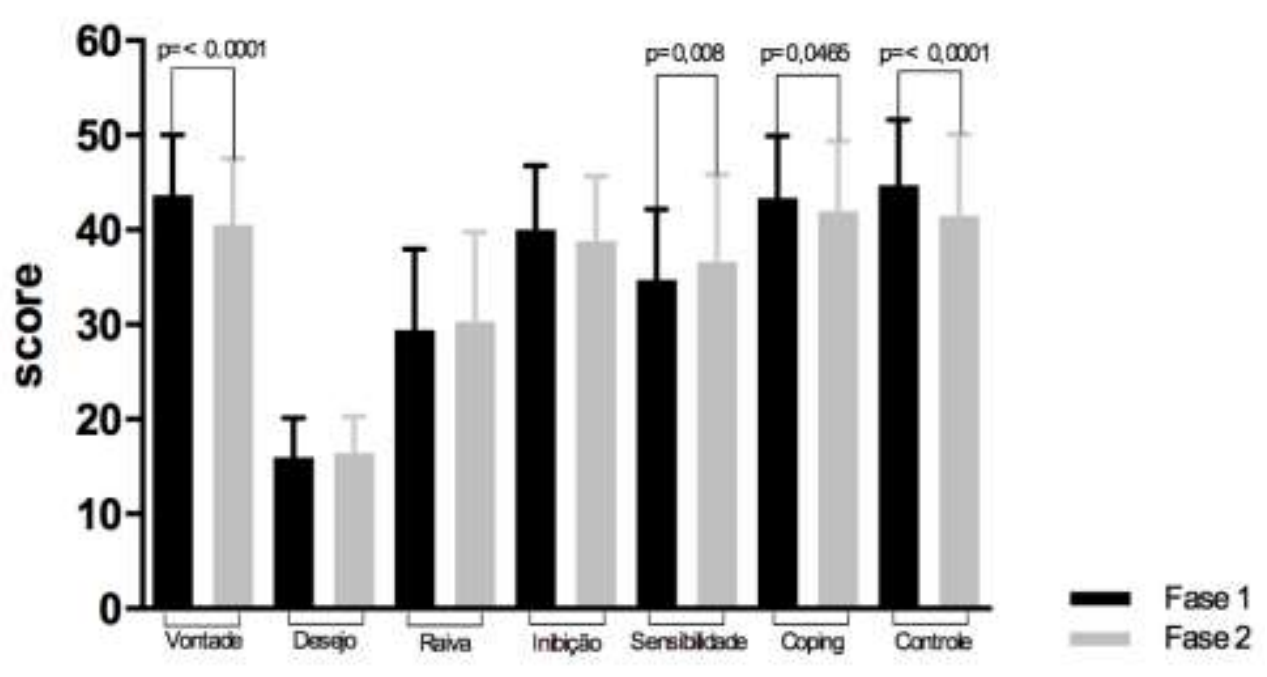

Fonte: Elaborado de acordo com os dados da pesquisa.

Gráfico 2: Funcionamento Emocional Global (GEF)

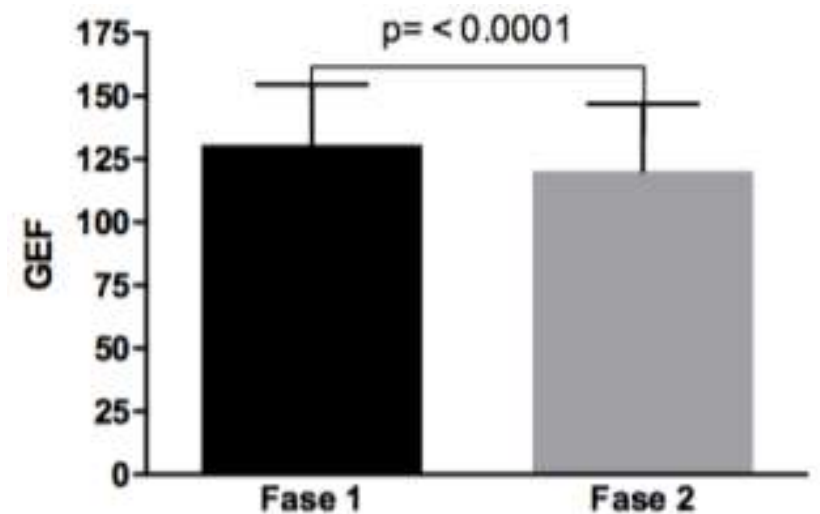

Fonte: Elaborado de acordo com os dados da pesquisa. 
Em relação à origem na avaliação emocional, apresentada na Tabela 1, analisa-se que o GEF sofreu alteração em estudantes que já habitavam o estado ( $\mathrm{p}=0.015$; coeficiente de incremento: -9.783 ; intervalo de confiança: -17.662 a -1.904 ). Entretanto, nesse contexto, quando analisados individualmente, os temperamentos emocionais não apresentaram tal resultado, com exceção da vontade ( $\mathrm{p}=0.014$; coeficiente de incremento: -2.771 ; intervalo de confiança: -4.969 a -0.573 ) e controle ( $\mathrm{p}=0.041$; coeficiente de incremento: -2.637; intervalo de confiança: -5.164 a -0.109). Ademais, o fato do discente ser proveniente de outro estado não influenciou significativamente o GEF e as dimensões emocionais.

Tabela 1: Relação do estado de origem com o temperamento emocional.

\begin{tabular}{|c|c|c|c|c|}
\hline & & Coeficiente de incremento & Intervalo de confiança & p-valor \\
\hline \multirow{9}{*}{ 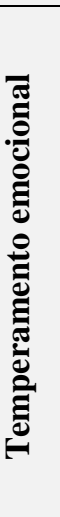 } & Vontade & & & \\
\hline & Não mudou & -2.771 & {$[-4.969 ;-0.573]$} & 0.014 \\
\hline & Mudou & -4.523 & -9.462 a 0.415 & 0.072 \\
\hline & Controle & & & \\
\hline & Não mudou & -2.637 & -5.164 a -0.109 & 0.041 \\
\hline & Mudou & -3.949 & -9.629 a 1.73 & 0.172 \\
\hline & GEF & & & \\
\hline & Não mudou & -9.783 & -17.662 a -1.904 & 0.015 \\
\hline & Mudou & -8.135 & -25.841 a 9.571 & 0.366 \\
\hline
\end{tabular}

Fonte: Elaborado de acordo com os dados da pesquisa.

Além disso, verificou-se que as pessoas do sexo feminino sofrerem maiores interferências nas dimensões vontade ( $\mathrm{p}=0.007$; coeficiente de incremento: -3.368 ; intervalo de confiança: -5.824 a -0.913 ) e sensibilidade ( $\mathrm{p}=0.024$; coeficiente de incremento: 3.106; intervalo de confiança: 0.413 a 5.799). As do sexo masculino, por sua vez, sofreram maior influência no controle ( $\mathrm{p}=0.028$; coeficiente de incremento: -4.359 ; intervalo de confiança: $-8.244 \mathrm{a}-0.474)$, sem influência significativa nas outras quatro dimensões emocionais (desejo, raiva, inibição e coping). O GEF apresentou associação significativa somente com o sexo feminino ( $\mathrm{p}=0.027$; coeficiente de incremento: -9.962 ; intervalo de confiança: -18.765 a -1.159 ), conforme exposto na Tabela 2 . 
Tabela 2: Relação do sexo com o temperamento emocional.

\begin{tabular}{|c|c|c|c|c|}
\hline & & Coeficiente de incremento & Intervalo de confiança & $\mathrm{P}$ \\
\hline \multirow{12}{*}{ 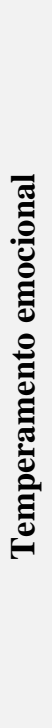 } & Vontade & & & \\
\hline & Feminino & -3.368 & $-5.824 \mathrm{a}-0.913$ & 0.007 \\
\hline & Masculino & -2.643 & -6.021 a 0.735 & 0.124 \\
\hline & Sensibilidade & & & \\
\hline & Feminino & 3.106 & 0.413 a 5.799 & 0.024 \\
\hline & Masculino & 0.093 & -3.611 a 3.797 & 0.960 \\
\hline & Controle & & & \\
\hline & Feminino & -2.077 & -4.901 a 0.747 & 0.148 \\
\hline & Masculino & -4.359 & $-8.244 \mathrm{a}-0.474$ & 0.028 \\
\hline & GEF & & & \\
\hline & Feminino & -9.962 & $-18.765 \mathrm{a}-1.159$ & 0.027 \\
\hline & Masculino & -8.568 & -20.678 a 3.542 & 0.164 \\
\hline
\end{tabular}

Fonte: Elaborado de acordo com os dados da pesquisa.

\subsection{Temperamento Afetivo}

Em uma avaliação global dos resultados, é perceptível o aumento da média dos padrões instável (7,46 para 8,01; p=0,0293) e externalizante (7,59 para 8,40; p=0,0002) (Gráfico 3). Ambos evidenciaram, nas duas fases, uma média que representa maior identificação com o comportamento e humor relacionados aos temperamentos, por parte dos alunos, pelo fato de ter ocorrido maior aproximação do valor máximo em comparação ao mínimo (Gráfico 3).

Além disso, foi verificada elevação significativa das médias de dois temperamentos que formam o externalizante: desinibido (2,46 para 2,72; p=0,0484) e eufórico (1,96 para 2,38; p=0,0003) (Gráfico 4).Em relação à origem, não se identificou relação estatística na avaliação das 12 dimensões do temperamento afetivo. Atrelado a isso, não houve relação significativa entre o sexo e a avaliação das 12 dimensões do temperamento afetivo.

Gráfico 3: Pontuação média dos padrões relacionados ao temperamento afetivo.

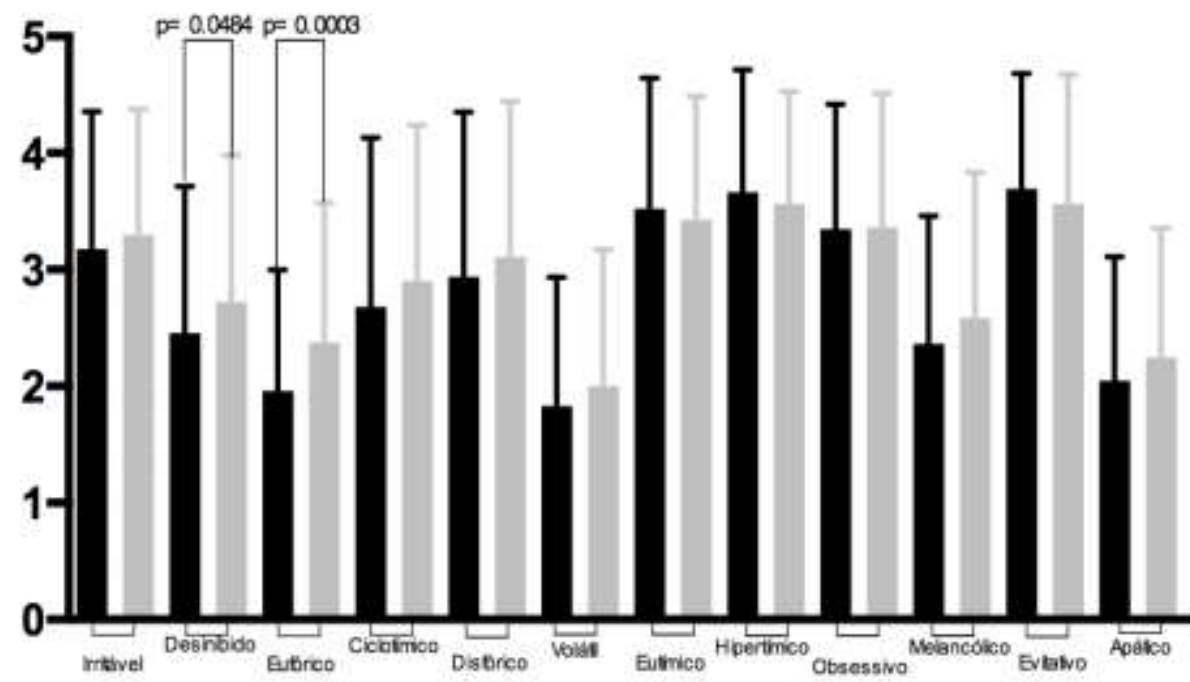

Fonte: Elaborado de acordo com os dados da pesquisa. 
Gráfico 4: Pontuação média de cada temperamento afetivo.

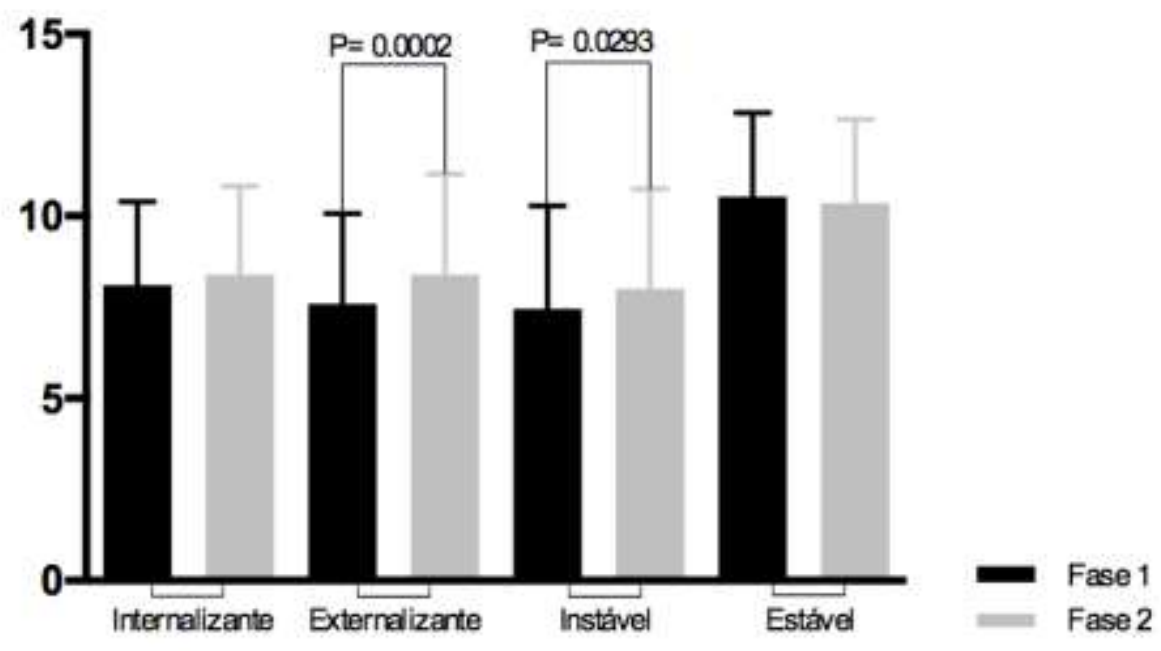

Fonte: Elaborado de acordo com os dados da pesquisa.

\section{Discussão}

A predominância da participação feminina denota maior número do referido sexo nos cursos de saúde da instituição de ensino analisada. Ainda, grande parte dos discentes é originada de outros estados do país. Em consonância com outros estudos, grande parte deles deixam a casa dos pais para dar continuidade à sua formação, e, apesar da perda do contato cotidiano com a família e das dificuldades advindas desse processo, as experiências adquiridas são vistas de forma positiva por estes indivíduos (Gottlieb et al., 2007; Teixeira et al., 2008).

Os autores Jang e Park (2016), ao considerarem a relação entre a personalidade, que está intimamente relacionada aos temperamentos (Lara, 2011), com o desempenho dos acadêmicos na ABP e com a aprendizagem colaborativa, demonstraram uma associação positiva entre a dimensão temperamental da persistência e a maior participação no trabalho em grupo, ou seja, os acadêmicos que possuíam uma maior perseverança eram mais ativos nas discussões de grupo. Contudo, a influência do método na evolução temperamental ainda permaneceu não investigada. Até o presente momento, o estudo aqui descrito foi o único que propôs esse tipo de avaliação.

Observa-se que a vontade está associada à persistência e iniciativa, representando uma abordagem pedagógica que visa a formação de profissionais possuidores de conhecimento, raciocínio, postura crítica e humanizada e de sujeitos sociais, o que possibilita a atuação em cenários complexos e a transformação da realidade (Fernandes et al., 2003). Foi verificada uma redução da dimensão vontade, o que leva a inferir que a ABP se associou de forma negativa com o temperamento, sendo o contrário ao esperado. Tal conjuntura pode estar associada aos estressores da metodologia, a qual rompe com o paradigma de ensino, à nova rotina acadêmica, ao estabelecimento de novas relações sociais e às atividades do cotidiano, afetando o comportamento e gerando situações que gastam energia sem o retorno recíproco (Margis et al., 2003; Paro; Bittencourt, 2013; Torres; Sampaio; Caldeira, 2019), o que poderia justificar a queda da média do temperamento. Em contrapartida, a experiência evolutiva da tristeza tem o objetivo de desenvolver a resiliência; por isso, aqueles com vontade alta e sensibilidade baixa, principalmente, buscam recursos para enfrentar situações difíceis (Lara, 2011). Um estudo concluiu que a ansiedade gerada pela metodologia ativa foi reduzida com o passar dos anos (Bento et al., 2017); dessa forma, pode-se levantar a hipótese que, apesar de a vontade ter reduzido sua média ao final do primeiro ano acadêmico, isso pode ser uma experiência, com o objetivo de gerar, no discente, mecanismos de enfretamento dos desafios e dificuldades.

Outro ponto relevante da aprendizagem colaborativa é o fato de ela estar presente quando os participantes 
compartilham responsabilidades de se dedicarem, previamente, aos estudos, para trazerem conhecimentos teóricos nas discussões do grupo, agregarem dados ao estudo prévio e contribuir para aprendizado de todos. Os debates saudáveis e a participação ativa dos acadêmicos são fatores importantes para a formulação de uma conclusão final do grupo referente à temática (Dolmans; Schmidt, 2006).

Os referidos aspectos da metodologia ativa de ensino podem se relacionar com o temperamento controle, em virtude de ele ser caracterizado pelo monitoramento do ambiente, persistência, responsabilidade, ordem e organização, capacidade de autorregulação, planejamento de estratégias e busca de adaptação ao meio (Lara, 2011). Porém, foi observada uma redução da média final na dimensão controle. Esta redução pode estar atrelada à vivência, por parte de alguns discentes, no final do primeiro ano, em um grupo tutorial que favoreceu a expressão de caracteres opostos aos que caracterizam o controle. $\mathrm{O}$ aprendizado, a harmonia e a produtividade do grupo podem ser comprometidos na presença de discussões desorganizadas, as quais estão presentes quando ocorre a tensão entre alunos dominantes e os passivos, de negligências com as etapas do método, das discussões superficiais e a ausência de conexões entre as ideias apresentadas pelo grupo, o que destoa do esperado para escores significativos do controle. É possível que tais fatores tenham sido frequentes nas turmas daqueles que manifestaram uma redução do temperamento (Diesel; Baldez; Martins, 2017).

Nos cursos da área da saúde, os impactos psicológicos são mais evidentes, devido às experiências com doenças, dor e sofrimento, necessidade de mudanças nas rotinas de estudos mais intensas e altas expectativas vindas de familiares e pacientes para os estudantes (Padovani et al., 2014). Concomitante, a extensa grade curricular obrigatória, a participação em atividades extracurriculares, o estabelecimento de relações sociais e o pouco tempo destinado ao sono favorecem o esgotamento físico e mental (Paro; Bittencourt, 2013). Diversos estudos realizados com estudantes de diferentes cursos da área da saúde evidenciaram a relação dos aspectos comentados com os altos níveis de estresse físico e psicológico (Murakami et al., 2019).

Nesse contexto, Lara (2011) destaca que a convivência com situações estressoras pode culminar no aumento da sensibilidade emocional. Esses fatos, talvez, expliquem o aumento da sensibilidade por parte dos discentes no final do primeiro ano letivo. Somado a isso, os diferentes cenários e competências exigidas pela metodologia ativa no processo de construção do aprendizado podem contribuir, de forma independente, para o aumento dos níveis de estresse (Bento et al., 2017) e, por conseguinte, da sensibilidade, no intuito de superar as dificuldades e desafios impostos (Paro; Bittencourt, 2013; Torres; Sampaio; Caldeira, 2019). A referida dimensão retrata o nível de reação individual às situações estressoras, como críticas, ofensas, rejeição, pressão, traumas, frustrações e perdas (Lara, 2011).

Segundo Tenório et al. (2016), o fato de a metodologia ativa estimular a autonomia e resolução de impasses, a partir de situações problemas, terminou por influenciar os alunos a buscarem soluções para as dificuldades presentes no seu curso. Tendo tais atitudes em vista, esperava-se um aumento da pontuação do temperamento coping pelos discentes, uma vez que tal dimensão reflete a capacidade de enfretamento adaptativo, resolução e aprendizado, ao se deparar com impasses e situações estressoras (Lara, 2011). Porém, houve um decréscimo da média da primeira para a segunda fase, sugerindo-se uma dificuldade dos alunos em contornar os desafios impostos pelo método e/ou desenvolver respostas adaptativas pertinentes acerca dos fatores estressores e a presença de um ajustamento geral negativo (Rudolph, Denning; Weisz, 1995).

Eventos positivos e negativos, quando suportáveis, possibilitam o desenvolvimento de estratégias de enfretamento para adaptação a futuros contextos (Lara, 2011; Antoniazzi; Dell'aglio; Bandeira, 1998). Logo, futuramente, os discentes poderiam apresentar um aumento do coping e, consequentemente, diminuição da sensibilidade, por alívio das situações estressoras. Bento et al. (2017) constataram uma redução dos altos níveis de ansiedade em estudantes, provavelmente causada pelo estresse da metodologia ativa, com o decorrer dos anos, refletindo uma adaptação à forma de ensino.

A redução do escore do GEF na segunda fase indica que a metodologia ativa e/ou o início da graduação em cursos da saúde promovem alteração desfavorável da configuração emocional, refletindo a ausência de adaptação ao novo cenário pelos 
alunos. A prática pedagógica está intimamente relacionada à autonomia, bem como ao desenvolvimento da consciência crítica, participação ativa, estímulo à curiosidade, indagação e criatividade (Freire, 2006; Mitre et al., 2008). Isso pode ser uma das explicações para o aumento significativo na pontuação do grupo externalizante entre as duas fases, denotando um maior reconhecimento por parte dos alunos com os comportamentos e estados de humor a ele relacionados, sendo indivíduos participativos, curiosos e criativos, na busca de sair de amarras cotidianas e convencionais (Bakker-Pieper; Vries, 2013; Lara, 2011), o que pode explicar o aumento significativo na pontuação do grupo entre as duas fases. Essas características apresentam relação com dois dos três temperamentos que o compõem - o desinibido e eufórico (Lara, 2011), e ambos apresentaram médias mais altas na segunda fase em relação à primeira.

Segundo Peduzzi e Agreli (2018), o trabalho em equipe é um artifício essencial para promover a integração das ações de saúde e organizar o serviço no âmbito da atenção primária do sistema de saúde brasileiro. Dessa forma, é possível que a metodologia ativa contribua para a formação de um maior número de profissionais aptos para o trabalho em equipe, por estimular comportamentos externalizantes, os quais podem estar envolvidos no desenvolvimento de habilidades comunicativas e colaborativas. Corroborando com esse pressuposto, Hulsman, Peters e Fabriek (2013) evidenciaram a existência de uma relação favorável entre a personalidade extrovertida e as competências comunicativas. Ainda mais, Guillén e Saris (2013) afirmaram que pessoas extrovertidas costumam ser sociáveis, além de se sentirem confortáveis na presença de outras pessoas e situações sociais variadas.

Além disso, os diferentes tipos de temperamento afetivo não implicam valor moral e, apesar de indivíduos eutímicos e hipertímicos serem considerados referências para estabilidade e adequação a diferentes contextos, os comportamentos e estados de humor relacionados as outras dimensões podem apresentar boa desenvoltura em novos ambientes, a depender do tipo e forma de interação exigidas (Lara, 2011). Os instáveis têm o padrão de iniciarem e logo desistirem de um projeto, assim se amoldam a contextos dinâmicos, com várias atividades curtas e com variabilidade de estímulos (Lara, 2011).

O aumento da média na dimensão instável observada neste estudo pode ser decorrente do aspecto dinâmico da proposta pedagógica no processo de ensino-aprendizagem, por incentivar a presença dos acadêmicos em diferentes cenários, além de propor aulas com debates, posicionamentos e questionamentos, a fim de se estabelecer uma conclusão conjunta (Diesel; Baldez \& Martins, 2017; Mitre et al., 2008).

Outras características associadas a dimensão instável também podem estar sendo desenvolvidas: o ciclotímico (humor imprevisível, alteração de fases entre entusiasmo, ansiedade, desânimo e perda de interesse); disfórico (simultaneidade das sensações de tensão, irritabilidade, ansiedade e agitação); e volátil (predominante em pessoas inquietas, dispersivas, desorganizadas, que têm dificuldades em concluir tarefas, muda de interesse rapidamente e, às vezes, são inconvenientes ou precipitadas) (Lara, 2011). Muitas dessas características poderiam influenciar de forma negativa no aprendizado colaborativo no grupo de tutorial e, como consequência, na formação profissional (Dolmans \& Schmidt, 2006).

\section{Considerações Finais}

Entendendo que as metodologias ativas são propostas de simulação dos problemas reais na formação de profissionais de saúde para melhor performance no campo de atuação, e que as experiências estudantis estão atreladas ao temperamento da pessoa como mediador no desempenho e nas relações, como também pode ser moldado a partir das vivencias na universidade, este estudo buscou avaliar o temperamento afetivo e emocional dos estudantes do primeiro ano na Universidade Federal de Sergipe no Campus de Lagarto-Sergipe, Brasil, que utiliza o método ABP. De modo resumido, houve impacto negativo no temperamento emocional dos estudantes nas dimensões vontade, controle, coping/resiliência, sensibilidade e GEF. Contudo, observou-se uma influência positiva nos aspectos de desinibição e euforia, consideradas importantes para a prática das atividades exigidas pela metodologia e no desempenho profissional. 
Porém, torna-se importante relacionar algumas limitações deste estudo, no qual foram incluídos estudantes de apenas uma universidade de metodologia ativa, inviabilizando a generalização dos resultados. Além disso, por ser uma pesquisa transversal, não é possível confirmar as relações de causa e efeito encontradas. Ainda, sabe-se que o primeiro ano da graduação é um período crítico para adaptação do discente, em virtude de representar uma etapa de transição, o que pode desencadear repercussões psicológicas e interferir no desempenho acadêmico. Tais fatos podem ter influenciado na evolução temperamental dos discentes, bem como as experiências de vida fora do âmbito acadêmico. Deste modo, considera-se importante a realização de mais estudos acerca do tema, trazendo mais evidências sobre a influência das metodologias de ensino ativas e tradicionais no temperamento afetivo e emocional de estudantes universitários.

\section{Referências}

Akiskal, H. S. (1998). Toward a definition of generalized anxiety disorder as an anxious temperament type. Acta Psychiatrica Scandinavica. Supplemen., 98, 66-73.

Antoniazzi, A. S. Dell'aglio, D. D. \& Bandeira, D. R. (1998). O conceito de coping: uma revisão teórica. Estud. Psicol, Natal. 3(2), $273-294$.

Bakker-Pieper, A., \& Vries, R. E. (2013). The Incremental Validity of Communication Styles Over Personality Traits for Leader Outcomes. Human Performance. 26, 1-19.

Beck, J. S. (2013) Terapia cognitivo-comportamental: teoria e prática. (2a ed.), Artmed.

Bento, L. M. A. et al. (2017). Percepção dos Alunos de Medicina quanto a Aprendizagem X Ansiedade na Metodologia Ativa. Rev. Ens. Educ. Cienc. Human., 18 (2), 78-182. https://seer.pgsskroton.com/index.php/ensino/article/view/4612/3586.

Berbel, N. A. N. (1995). Metodologia da Problematização: uma alternativa metodológica apropriada para o Ensino Superior. Semina. Cio Soc./Hum, 16(2), 919.

Castano-Perez, G. A. \& Calderon-Vallejo, G. A. (2014). Problemas associados ao consumo de álcool em estudantes universitários. Rev. Latino-Am. Enfermagem, 22(5), 739-46.

Cloninger, C. R., Svrakic, D. M. \& Przybeck, T. R. (1993). A psychobiological model of temperament and character. Archivesof General Psychiatry, 50(12), 975-990. http://jamanetwork.com/journals/jamapsychiatry/articleabstract/496431.

Diesel, A., Baldez, A. L. S. \& Martins, S. N. (2017). Os princípios das metodologias ativas de ensino: uma abordagem teórica. Revista Thema. 14(1), 268-288. http://dx.doi.org/10.15536/thema.14.2017.268-288.404.

Dolmans, D. H. \& Schmidt, H. G. (2006). What do we know about cognitive and motivational effects of small group tutorials in problem-based learning? Adv. Health Sci. Educ. TheoryPract., 11, 4, 321-336.

Ekman, P. (2011). A linguagem das emoções: Revolucione a sua comunicação e seus relacionamentos reconhecendo todas as expressões das pessoas ao redor. Tradução Carlos Szlak. Lua de Papel.

Fernandes, J. D. et al. (2003). Estratégias para a implantação de uma nova proposta pedagógica na escola de enfermagem da Universidade Federal da Bahia. Revista Brasileira de Enfermagem. 56(4).

Gottlieb, B.H et al. (2007). Tipos e precipitadores de crescimento e declínio na idade adulta emergente. Journal of Adolescent Research. 22(2), $132-155$.

Guillén, L. \& Saris, W. E. (2013). Competencies, Personality Traits, and Organizational Rewards of Middle Managers: A Motive-Based Approach. Human Performance, 26, p. 66-92.

Hendry, G. D., Ryan, G. \& Harris, J. (2003). Group problems in problem-based learning. Medical Teacher, 25(6), 609-616.

Hulsman, R. L., Peters, J. F., Fabriek, M. (2013). Peer-assessment of medical communication skills: e impact of students' personality, academic and social reputation on behavioural assessment. Patient. Educ. Couns., 92, 346-354.

INEP. Resumo técnico: Censo da Educação Superior 2015 (2018). Brasília: Instituto Nacional de Estudos e Pesquisas Educacionais Anísio Teixeira, (2a ed.).

Jang, H. W. \& Park, S. W. (2016). Effects of personality traits on collaborative performance in problem-based learning tutorials. Saudi Med J., 37(12), 13651371 .

Lara, D. R. et al. (2012) The Affective and Emotional Composite Temperament (AFECT) model and scale: A system-based integrative approach. JournalofAffectiveDisorders, 140(1), 14-37.

Lara, D. (2011). Temperamento e humor: uma abordagem integrada da mente. Observatório Gráfico.

Margis, R. et al. (2003). Relação entre estressores, estresse e ansiedade. Rev. Psiquiatr., 25, 64-74. http://dx.doi.org/10.1590/S0101-81082003000400008 
Research, Society and Development, v. 10, n.6, e17110615635, 2021

(CC BY 4.0) | ISSN 2525-3409 | DOI: http://dx.doi.org/10.33448/rsd-v10i6.15635

Mccrae, R. R., \& Costa, P. T. (1987). Validation of the five-factor model of personality across instruments and observers. Journal of Personality and Social Psychology, 52(1), 81-90.

Mcgue, M., Slutske, W., Taylor, J. \& Iacono, W. G. (1997). Personality and substance use disorders: I. Effects of gender and alcoholism subtype. Alcoholism: Clinical and Experimental Research, 21, 513-520.

Mitre, S. M. et al. (2008). Metodologias ativas de ensino-aprendizagem na formação profissional em saúde: debates atuais. Ciência e Saúde Coletiva, 13(2).

Mpofu, D. J. S., Das, M. \& Stewart, T. (1998). Perceptions of group dynamics in problem-based learning sessions: a time to reflect on group issues. Medical Teacher, 20(5), 421-427.

Murakami, K. et al. (2019). Estresse psicológico em estudantes de cursos de graduação da área da saúde: subsídios para promoção de saúde mental. Rev. Med., 98(2), 108- 13 .

Neufeld, C. B., Ferreira, I. M. F., Caetano, K. A. S. \& Versuti, F. M. (2020). Aprendizagem Baseada em Problemas: estudo exploratório da percepção de estudantes de Psicologia. Research, Society and Development, 9(5), e48952109. https://rsdjournal.org/index.php/rsd/article/view/2109.

Padovani, R. C. et al. (2014). Vulnerabilidade e bem-estar psicológicos do estudante universitário. Rev. Bras. Ter. Cogn., 10(1), 2-10.

Paro, C.A. \& Bittencourt, Z. Z. L. C. (2013). Qualidade de Vida de Graduandos da Área da Saúde. Revista Brasileira de Educação Médica. 37(3), 365-375.

Pereira, A. S. et al. (2018). Metodologia da Pesquisa Científica. UFSM.

Reis, R.G. (2015). Consumo de álcool, personalidade e ajustamento emocional em estudantes universitários. Universidade Lusófona de Humanidades e Tecnologias, Escola de Psicologia e Ciências da Vida. Lisboa.

Rios, P. A. A. et al. (2008). Consumo e uso abusivo de bebidas alcoólicas em estudantes universitários do município de Jequié/BA. Rev.Saude.Com., 4(2), 105-116.

Rudolph, K. D., Denning, M. D. \& Weisz, J. R. (1995). Determinants and consequences of children's coping in the medical setting conceptualization, review, and critique. Psychological Bulletin, 118(3), 328-357.

Saupe, R. et al. (2011). Competência dos Profissionais da Saúde para o Trabalho Interdisciplinar. Interface comun. saúde educ., 9(18), 521-536.

Silva, E. C. \& Tucci, A. M. (2018). Correlação entre ansiedade e consumo de álcool em estudantes universitários. Revista Psicologia: Teoria e Prática, 20(2), 93-106.

Silva, J. N. et al. (2015). Consumo de álcool entre universitários. RBP e CS. 2(2), 35-40.

Teixeira, M. A. P. et al. (2008). Adaptação à universidade em jovens calouros. Psicol. Esc. Educ. (Impr.). 12(1), 185-202.

Tenório, L. P. et al. (2016). Saúde Mental de Estudantes de Escolas Medicas com Diferentes Modelos de Ensino. Revista Brasileira de Educacao Medica, 40(4), 574- 582

Torres, V., Sampaio, C. A. \& Caldeira, A. P. (2019). Ingressantes de cursos médicos e a percepção sobre a transição para uma aprendizagem ativa. Interface. 23.

Ventura, R. C. M. O., Souza, R. A., Mendes, A. A., Altino Filho, H. V., Trindade, F. C., Araujo, G. L. \& Pôncio, T. G. H. O. (2020). Metodologias Ativas e Participativas: uma análise da produção do UNIFACIG no Fórum STHEM-BRASIL. Research, Society and Development, 9(7), e425973648. https://rsdjournal.org/index.php/rsd/article/view/3648.

Virtanen, P. J. et al. (1999). What happens in PBL tutorial sessions? Analysis of medical students' written account. Medical Teacher, 21(3), 270-276. 